\title{
THE
}

5-1-1985

\section{Surface Free Energies of Three-Dimensional Ising Models and Universality of Finite-Size Scaling Amplitudes by Direct Monte Carlo Sampling}

\author{
K. K. Mon \\ M. P. Nightingale \\ University of Rhode Island, nightingale@uri.edu
}

Follow this and additional works at: https://digitalcommons.uri.edu/phys_facpubs

Terms of Use

All rights reserved under copyright.

\section{Citation/Publisher Attribution}

Mon, K. K., \& Nightingale, M. P. (1985). Surface free energies of three-dimensional Ising models and universality of finite-size scaling amplitudes by direct Monte Carlo sampling. Physical Review B, 31(9), 6137-6139. doi: 10.1103/PhysRevB.31.6137

Available at: http://dx.doi.org/10.1103/PhysRevB.31.6137

This Article is brought to you for free and open access by the Physics at DigitalCommons@URI. It has been accepted for inclusion in Physics Faculty Publications by an authorized administrator of DigitalCommons@URI. For more information, please contact digitalcommons-group@uri.edu. 


\title{
Surface free energies of three-dimensional Ising models and universality of finite-size scaling amplitudes by direct Monte Carlo sampling
}

\author{
K. K. Mon \\ Department of Physics, University of Rhode Island, Kingston, Rhode Island 02881 \\ M. P. Nightingale \\ Department of Physics, University of Rhode Island, Kingston, Rhode Island 02881 \\ and Department of Physics, Massachusetts Institute of Technology, \\ Cambridge, Massachusetts 02139 \\ (Received 13 March 1985)
}

\begin{abstract}
Direct Monte Carlo sampling is employed to obtain estimates of excess surface free energies of threedimensional Ising models at criticality. Results for simple-cubic and body-centered-cubic lattices provide strong evidence for the universality of finite-size scaling amplitudes and in particular the related interaction per unit area, mediated via the incipient long-ranged correlations, of two free surfaces a finite distance apart.
\end{abstract}

The universality of critical amplitude ratios has been studied quite extensively in the literature. ${ }^{1}$ The observation that certain finite-size amplitudes in themselves are universal is fairly recent. ${ }^{2-5}$ For two dimensions Cardy has derived some of these amplitudes from conformal invariance. $^{2}$ Also, the universality of these quantities has been argued on the basis of renormalization-group ${ }^{3}$ and general scaling arguments. ${ }^{4}$ Universality has been verified numerically in various two-dimensional models. ${ }^{3,4,5}$ Although the renormalization-group and scaling arguments also apply in three dimensions we know of no prior numerical evidence in favor of the aforementioned universality.

In this Rapid Communication we report the results of a scaling analysis of surface free energies of finite threedimensional systems with free surfaces. The results were obtained employing a Monte Carlo simulation technique developed recently. ${ }^{6}$ The calculations were done as a function of aspect ratio, which measures the system size perpendicular to the free surface in units of the size parallel to it. The results support the universality of the corresponding scaling function which describes the finite-size behavior of the excess free energy of a free surface in different threedimensional Ising models. This is important in itself from a theoretical point of view, being the first numerical evidence in support of the universality of finite-size amplitudes in three dimensions. Experimentally it is of particular interest if the validity of this result extends, as we expect it does, throughout the whole Ising universality class. In that case, the force constant measuring, as a function of distance, the interaction per unit area of two plates immersed, e.g., in a critical binary mixture is universal if expressed in units of $k T{ }^{7}$ Further, it was suggested recently that a related (see below) constant governs the behavior of the thickness of a (near-) critical wetting layer that may intrude between two noncritical phases. ${ }^{8}$ An example of the latter is a ternary mixture at a critical end point in the vicinity of the tricritical point, if wetting is such that the critical phase excludes the spectator phase from contact with the wall. Also from a computational point of view, the results reported in this $\mathrm{Ra}$ pid Communication are interesting. They show that data obtained by direct Monte Carlo sampling, and analyzed by finite-size scaling methods, yield very accurate estimates of the free energies of free surfaces of the Ising models considered. Apart from a real-space renormalization-group calculation, to our knowledge, these are the first numerically exact estimates. ${ }^{9}$ The ability to calculate surface free energies for lattice systems accurately by direct Monte Carlo sampling is of interest to a wide range of important physical problems. $^{10}$

We have performed calculations for finite Ising models defined on various lattices: simple square, and triangular in two dimensions (to test the method), and simple cubic (sc) and bcc in three dimensions. In general, we consider a square hyperprism in $d$ dimensions, with sides of the following lengths (measured in units of the lattice constant $a$ ): $n_{\perp}$ along one, and $n_{\|}$along the remaining $d-1$ perpendicular directions. For a full treatment of finite-size scaling theory for surfaces, and analytical calculations checking its validity we refer to a paper ${ }^{11}$ by Au-Yang and Fisher, where also further references to the literature can be found. A brief summary is sufficient for our purposes.

The total free energy of a system with periodic boundary conditions in all directions is denoted $F ; F^{\mathrm{x}}$ is the same quantity if the boundary conditions are periodic in all but the $n_{\perp}$ direction, in which they are free. The surface free energy per unit area of the two $d-1$ dimensional free surfaces is $f^{\mathrm{x}}=\left(n_{\|} a\right)^{1-d}\left(F^{\mathrm{x}}-F\right)$. Its singular part $f_{\text {sing }}^{\mathrm{x}}$ satisfies the scaling relation

$f_{\text {sing }}^{\mathrm{x}}\left(t, 1 / n_{\perp}, 1 / n_{\|}\right)=(b a)^{1-d} f_{\text {sing }}^{\mathrm{x}}\left(b^{y_{\mathrm{T}}} t, b / n_{\perp}, b / n_{\|}\right)$,

where $t=\left(T-T_{c}\right) / T_{c}$, the temperature deviation away from the critical point. Note that at the critical point the difference of $f^{\mathrm{x}}$ and $f_{\text {sing }}^{\mathrm{x}}$ is precisely the critical surface free energy $f_{\infty}^{\mathrm{x}}$. Since $b$ is an arbitrary rescaling length it follows that at $t=0$ the surface free energy is given in terms of a scaling function as follows:

$$
f_{\text {sing }}^{\mathrm{x}}\left(0,1 / n_{\perp}, 1 / n_{\|}\right)=\left(n_{\perp} a\right)^{1-d} \Delta\left(n_{\perp} / n_{\|}\right) .
$$

Within the statistical uncertainty of our calculations this scaling function $\Delta$ is a universal function of the aspect ratio $n_{1} / n_{\|}$. If $Y$ is defined as the finite-size scaling amplitude of the bulk free energy $F$ for a system of vanishing aspect ratio, then the universal number $\Delta(0)+Y$ is experimentally 
accessible via the experiments mentioned above. ${ }^{8}$

Note that - a minimum requirement for universality - the scaling function $\Delta$ is invariant under a redefinition of the unit of length associated with the lattice spacing $a$. For an anisotropic system one should expect a universal scaling function to emerge if different units of length are used in different directions. These units, which define at most $d-1$ nonuniversal ratios, restore full spherical symmetry to the surfaces of constant correlation at large distances. For the square lattice this result was verified analytically. ${ }^{12}$

The systems considered have reduced Hamiltonians-a factor $-1 / k T$ is absorbed-of the form

$$
H\left(K_{\perp}\right)=K \sum_{(i, j)} s_{i} s_{j}+K_{\perp} \sum_{(i, j)} s_{i} s_{j},
$$

where the first summation is over all nearest-neighbor pairs of sites $(i, j)$ except those that cross an arbitrary plane perpendicular to the $n_{\|}$direction; the latter feature in the second sum. For $K_{1}=K$ the Hamiltonian describes the fully periodic system; for a vanishing surface interaction $K_{\perp}$ the free surface is obtained. The interaction $K$ is set at the bulk critical value $K_{c}$.

We denote by $Z\left(K_{\perp}\right)$ the partition function of a system with Hamiltonian (3). For the special cases $K_{\perp}=K$ (periodic) and $K_{1}=0$ (free) we use, respectively, $Z$ and $Z^{\mathrm{x}}$. For the surface free energy one computes

$$
F^{\mathrm{x}}-F=-\ln \left(Z^{\mathrm{x}} / Z\right) .
$$

For small systems the ratio of the partition functions can be calculated employing the expression

$$
Z^{x} / Z=\left\langle e^{H(K)-H(0)}\right\rangle_{K},
$$

where the thermal average \langle\rangle is labeled by the surface interaction that determines the Hamiltonian and corresponding Boltzmann weights. An expression requiring two separate Monte Carlo processes for its evaluation, but with the advantage of reduced variance needed for the larger systems, is the following

$$
Z^{\mathrm{x}} / Z=\langle g(H(0)-H(K))\rangle_{K} /\langle g(H(K)-g H(0))\rangle_{0},
$$

where $g(x)=1 /\left(1+e^{x}\right){ }^{13}$ For the largest systems we considered, even expression (6) was inappropriate. In those cases multistage sampling was employed to calculate separately the thermal averages according to Eq. (5) in

$$
Z^{\mathrm{x}} / Z=\left[Z(0) / Z\left(K_{1}\right)\right]\left[Z\left(K_{1}\right) / Z(K)\right],
$$

where $K>K_{1}>0$. Obviously, this expression can be generalized to any sequence of intermediate systems with boundary conditions varying smoothly between free and periodic We used typically 60000 Monte Carlo steps per spin per stage in calculating the surface free energies.

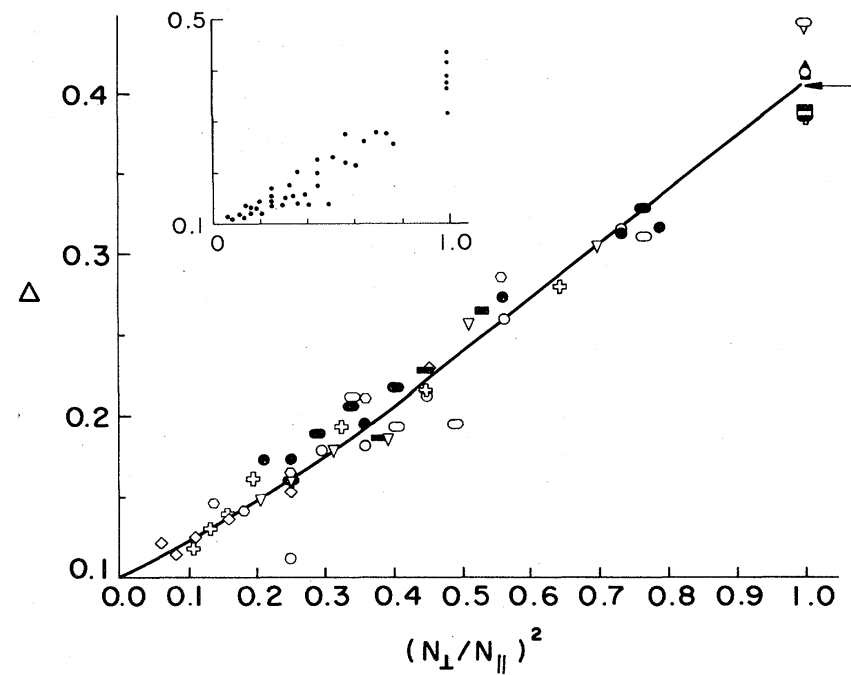

FIG. 1. The finite-size scaling function as function of the aspect ratio squared. The data for the simple cubic and body-centeredcubic lattices are indicated by full and open symbols, respectively. Data are displayed for the various values of $n_{\|}: n_{\|}=2$ (diamonds), $n_{\|}=3$ (hexagons), $n_{\|}=4$ (crosses), $n_{\|}=5$ (inverted triangles), $n_{\|}=6$ (circles), $n_{\|}=7$ (ovals), $n_{\|}=8$ (rectangles), $n_{\|}=9$ (squares), and $n_{\|}=10$ (triangles). The arrow indicates the average value of $\Delta(1)$, as obtained from both lattices. The dimensionless critical (double) surface free energies $f_{\infty}^{\mathrm{x}}$ were set to 0.03724 (simple cubic) and 0.07988 (body-centered-cubic). The inset illustrates the sensitivity of the scaling plot to the critical surface free energy: for the body-centered-cubic lattice $f_{\infty}^{\mathrm{x}}$ was changed to 0.08105 .

To test the method we first performed calculations on the square and triangular systems in two dimensions. We obtained $f_{\infty}^{\mathrm{x}}=0.1817 \pm 0.002$ and $0.224 \pm 0.007$, respectively, which, within the error bars, is in agreement with exact calculations for the square lattice. ${ }^{11}$

Having established that the method works, we discuss the data obtained for the sc and the bcc lattices. In a scaling plot the surface free energy of the system in the thermodynamic limit (i.e., the regular part of the free energy) is the only fitting parameter used to collapse the data onto a single curve. Of course this surface free energy is lattice dependent, being roughly proportional to the number of broken bonds. Figure 1 shows, not only do the data collapse for the two lattices separately as predicted by scaling theory, but more importantly the two lattices are indistinguishable within the accuracy of our calculations: a striking illustration of the universality of the finite-size amplitude. In the inset of Fig. 1 the sensitivity of the scaling procedure to the value of the surface free energy is illustrated. Using the extent to which the data collapse as a measure of the er-

TABLE I. Summary of Monte Carlo results for the three-dimensional Ising models. The critical couplings for the simple cubic (sc) and body-centered-cubic (bcc) lattices were taken from Ref. 14. Note that we calculated the excess surface free energy of two free surfaces.

\begin{tabular}{llccc}
\hline \hline & \multicolumn{1}{c}{$K_{c}$} & \multicolumn{1}{c}{$f_{\infty}^{\mathrm{x}}$} & $\Delta(0)$ & $\Delta(1)$ \\
\hline sc & 0.2217 & $0.03724 \pm 0.0006$ & $\sim 0.1$ & $0.4024 \pm 0.016$ \\
bcc & 0.15737 & $0.07947 \pm 0.0008$ & -0.1 & $0.410 \pm 0.03$ \\
\hline \hline
\end{tabular}


ror in the result we obtain the estimates of the surface free energies displayed in Table $I^{15}$ There we also summarize the values obtained for the force constants $\Delta(0)$ and $\Delta(1)$. Note that the estimate of $\Delta(0)$ is also subject to the uncertainty associated with the extrapolation of the scaling function to zero.

In conclusion, we have shown that direct Monte Carlo sampling provides an accurate means of calculating surface free energies at criticality. Further, while these free ener- gies are nonuniversal, our results strongly support that their corrections due to a finite system size are universal.

It is our pleasure to acknowledge valuable discussions with J. O. Indekeu. We should also like to thank John Rehr and Michael George for sending us their results prior to publication. This work was supported by the National Science Foundation, Contract No. DMR-8406186, and by a grant from the Research Corporation.
${ }^{1}$ See, for example, A. Aharony and P. C. Hohenberg, Phys. Rev. B 13, 3081 (1976); H. Tarko and M. E. Fisher, ibid. 11, 1217 (1975).

2J. L. Cardy, J. Phys. A 17, L385 (1984).

${ }^{3}$ M. P. Nightingale and H. W. J. Blöte, J. Phys. A 16, L657 (1983).

${ }^{4}$ V. Privman and M. E. Fisher, Phys. Rev. B 30, 322 (1984).

${ }^{5}$ B. Derrida and J. de Sèze, J. Phys. (Paris) 43, 475 (1982); J. M. Luck, J. Phys. A 15, L169 (1982); J. L. Pichard and G. Sarma, J. Phys. C 14, L617 (1981); K. A. Penson and M. Kolb, Phys. Rev. B 29, 2854 (1984); H. W. J. Blöte and M. P. Nightingale, Physica A 129, 1 (1985); T. W. Burkhardt and I. Guim (unpublished).

${ }^{6}$ K. K. Mon and D. Jasnow, Phys. Rev. A 30, 670 (1984); Phys. Rev. A (to be published); K. K. Mon (unpublished).

${ }^{7}$ M. E. Fisher and P.-G. de Gennes, C. R. Acad. Sci. 287, 207 (1978).

${ }^{8}$ M. P. Nightingale and J. O. Indekeu, Phys Rev. Lett. 54, 1824 (1985).

${ }^{9}$ Series expansions for the free energy of infinite systems of finite thickness are available [G. A. T. Allan and M. E. Fisher (unpub- lished work)], but they have not actually been employed to obtain the excess surface free energy; N. M. Svrakić, R. Pandit, and M. Wortis, Phys. Rev. B 22, 1286 (1980).

${ }^{10}$ See, for recent reviews, Applications of Monte Carlo Methods, edited by K. Binder (Springer, Berlin, 1984).

${ }^{11}$ H. Au-Yang and M. E. Fisher, Phys. Rev. B 11, 3469 (1975); 21, 3956 (1980); M. E. Fisher and H. Au-Yang, Physica A 101, 225 (1980). To our knowledge, no exact results have been reported for the triangular lattice.

12J. O. Indekeu, M. P. Nightingale, and V. Wang (unpublished).

${ }^{13}$ C. H. Benett, J. Comput. Phys. 22, 245 (1976).

${ }^{14} \mathrm{M}$. George and J. J. Rehr (unpublished); R. B. Pearson, J. L. Richardson, and D. Toussaint, Phys. Today 36, 52 (1983); also see G. S. Pawley, R. H. Swendsen, D. J. Wallace, and K. G. Wilson, Phys. Rev. B 29, 4030 (1984).

${ }^{15}$ The renormalization-group estimate of the surface free energy is of Šrakic et al. ${ }^{9}$ for the sc lattice is of the same order of magnitude as ours. 